\title{
A Case Study for a New Peer-Review Journal on Race and Ethnicity in American Higher Education
}

\author{
Cristobal Salinas Jr. \\ Educational Leadership and Research Methodology, Florida Atlantic University, 777 Glades Road, ED47-242, \\ Boca Raton, FL 33431, USA; salinasc@fau.edu
}

Received: 13 March 2018; Accepted: 29 May 2018; Published: 4 June 2018

\begin{abstract}
In this exploratory case study, the interests, attitudes, and opinions of participants of the National Conference on Race and Ethnicity (NCORE) in American Higher Education are presented. This case study sought to understand how college and university administrators and faculty perceived the need to create a peer-reviewed journal that aimed to support and create opportunities to publish research, policy, practices, and procedures within the context of race and ethnicity in American higher education. The findings of this study reflect that the vast majority of those surveyed $(n=605)$ and interviewed $(n=5)$ support, and are interested in, having a peer-reviewed journal that focuses on race and ethnicity in American higher education.
\end{abstract}

Keywords: race and ethnicity; scholarship; higher education; open-access; faculty of color

\section{Introduction}

SCImago Journal \& Country Rank and Directory of Open Access Journals confirmed that there is a lack of journals with a primary focus on research related to race and ethnicity, and only one international journal aims primarily to publish scholarship with a focus on race and ethnicity in education [1,2]. Although many peer-review journals use the terms diversity, multiculturalism, social justice, and inclusion in an effort to demonstrate a commitment to these words [3], there is still a need for peer-review journals that focus on race and ethnicity. A new journal on race and ethnicity can create new publishing opportunities for Faculty of Color that produce and focus on this area of scholarship, as often times they have been rejected by mainstream and top-tier journals.

The body of literature illustrates that Faculty of Color in the United States are more likely than White Faculty to produce scholarship with a focus on race and ethnicity themes, including gender inequities, diversity, and social justice [4-7]; yet, there is a lack of peer-reviewed journals that focus solely on publishing scholarship with aims and scope on race and ethnicity. Moreover, data collected for this case study show that there is the need to create additional publishing outlets and peer-review journals that solely focus on the production and dissemination of scholarship of race and ethnicity in American higher eduation. A new peer-review journal with aims and scope to publish scholarship on race and ethnicity in American higher education could support Faculty of Color, who are mostly socialized by White Faculty members and expected by their institutions to "seek out top-tier journals for publishing their research" ([6], p. 15). Faculty of Color are more likely than White Faculty to "produce scholarship that addresses different social inequalities, often through the fields of humanities, education, social sciences, and ethnic studies ..." and their "scholarship is often undervalued by the academic profession" ([5], p. 171).

American higher education needs more peer-reviewed journals that acknowledge the "cultural resources that are based on the epistemologies that many faculty of color bring to academia" through their scholarship and lived experiences ([5], p. 172). Therefore, the purpose of this case study was to evaluate the interests, attitudes, and opinions of participants of the National Conference on Race and 
Ethnicity (NCORE) in American Higher Education regarding the need to create a peer-reviewed journal that aims to support and create opportunities to publish research, policy, best practices, and procedures with an emphasis on race and ethnicity in American higher education. This case study adds to the relatively small, although growing, body of literature on scholars and practitioners' interests, attitudes, and opinions in publishing in peer-reviewed journals that center race and ethnicity to deconstruct the master narratives and epistemologies, and offer an alternative scholarship to educational research.

The literature described Faculty of Color's experiences as feelings of intellectual isolation due to the lack of mentoring and research support, and the impact of peer-reviewed journals [7]. After the review of the literature, I describe the impact of the lack of race and ethnicity of peer-reviewed journals in American higher education. I then describe the conceptual framework and research design, including data collection, data analysis, and the findings of this study. The final sections offer a discussion and overview of the developments of this case study.

\subsection{Faculty of Color}

While Faculty of Color have been studied frequently in the last twenty years [5,8,9], more research is needed to explore why Faculty of Color remain underrepresented at universities and their superb work remains practically unnoticed. Scholars have identified that Faculty of Color in higher education experience several challenges to their success of recruitment, retention, and promotion and tenure. For example, Faculty of Color experience a lack of mentorship; a lack of support for research and publications; and a lack of information about tenure and promotion, isolation, and cultural taxation $[4,7,8,10]$. Jayakumar et al. stated that "intellectual isolation affects research accomplishments and contributes ... little to no access to information" on the process to tenure advancement ([7], p. 542). The lack of support creates occupational stress among Faculty of Color, but also, they have heavy service loads with less time for research that leads to the rewards of tenure and promotions. Despite the ongoing challenges Faculty of Color face in American higher education, they play an important and unique role in today's educational system and society.

Villalpando and Delgado Bernal indicated that Faculty of Color were as productive as White Faculty in research and publications, and spent more time preparing for and teaching courses and advising students [11]. Yet, Faculty of Color experience less support for their research and teaching, and they have heavy service loads with less time for research that leads to the rewards of tenure and promotion $[4,12,13]$. Faculty of Color are more likely to produce research on issues of race, ethnicity and gender inequities, diversity, and social justice, whereas White Faculty are often asked to produce according to the institutions' research agenda and values [4,7]. When Faculty of Color produce scholarship that focuses on race, ethnicity, and gender inequities, it is more challenging to publish it in mainstream journals, as mainstream and top-tier journals do not focus on topics of race and ethnicity [6]. Therefore, a peer-reviewed journal that focuses on race and ethnicity is necessary to support Faculty of Color in their advancement to tenure and promotion.

\subsection{Overview of the Impact of Peer-Reviewed Journals}

In the 21st century, academic journals played a significant role in scholarly communities that serve to build a collective knowledge base, communicate information, validate the quality of research, distribute rewards when scholars are evaluated, and build scientific communities [14]. In addition, Francis affirmed academic journals keep up-to-date on research in the field; provide credit towards promotion or tenure for faculty; offer experience to improve personal research, thinking, and writing; and provide support in the field [15].

Publishing is important and demanding for the progress of scholars' research [16]. In their work, Bray and Major indicated that publications in peer-reviewed journals are the most recent advances in knowledge, and "the process of getting published in the "right" journals is critical to faculty success" ([17], p. 2]. Publishing is characterized for faculty members as conforming to the status quo, as well as promotion to get tenure, and a potential salary increase depending on the institution and department 
policies [18-20]. Peer-reviewed journals have formed a culture in higher education that promotes important elements in promotion and tenure [4,15,21,22]; yet, scholarship produced by Faculty of Color in humanities, education, social sciences, and ethnic studies, "is often undervalued by the academic profession" ([5], p. 171).

Researchers and scholars have created hegemony in the tradition of the peer-review publishing process, as they maintain traditional paradigms for manuscripts as research and scholarship confronts the hegemony of tradition [23]. Thomas and Schmidt indicated that to be considered a rigorous scholar and researcher, an individual has to embrace the traditional peer-review publishing process and consider the weight of authorship [23]. To write in a scholarly manner as a single author is considered to be rigorous and an act of isolation; when publishing in collaboration, the first or second author is determined based on the weight of who carried out the majority of the research, writing, and editing. This status quo culture has led academics to "think of journals as a social institution" and social hierarchy ([24], p. 9), and has created challenges for some faculty members, higher education practitioners, and administrators to publish in peer-reviewed journals [16].

\subsection{Absence of Race and Ethnicity Peer-Reviewed Journals}

As stated earlier, SCImago Journal \& Country Rank and Directory of Open Access Journals confirmed that there is a lack of journals that aim to publish scholarship on research concerning race and ethnicity [1,2]. SCImago Journal \& Country Rank confirmed that from the total of 20,544 subscription journals [1], and the Directory of Open Access Journals confirmed that from the 9929 open-access journals, there is only one British journal, Race Ethnicity in Education, whose aims and scope are to publish scholarship on race and ethnicity in education [2]. It is important to note that both SCImago Journal \& Country Rank and Directory of Open Access Journals established that there are over 150 journals that focus on publishing scholarships exclusively on a particular race or ethnicity (i.e., Asian Ethnicity, Journal of Black Studies, Journal of Hispanic Higher Education), none of which focus on publishing scholarships that are inclusive of all races and ethnicities [1,2]. Even though mainstream journals published some scholarly research on race and ethnicity, there is a lack of this scholarship and research in academic peer-reviewed journals that solely focuses on the production and dissemination of scholarship of race and ethnicity in American higher education.

Researchers and scholars have been dedicated to the advancement of higher education through the production and dissemination of scholarship through various academic journals. Peer-reviewed journals create opportunities for the distribution of research, and influence other journals to focus on different issues and topics associated with higher education, such as college student development, faculty, and staff, as well as the specific types of institutions of higher learning, including two-year and four-year institutions. However, most peer-reviewed journals in higher education's aims and scope do not solely focus on the production and dissemination of scholarship on race and ethnicity in American higher education. Rather, their scholarship focuses on culture, organization, research, leadership, professional development, history of higher education, and academia, sometimes without acknowledging the need for racially and ethnically diverse students, faculty, administrators, social justice, multiculturalism, inclusivity, and diversity. It was not until the 1980s that articles in peer-reviewed journals started to focus on broader interest in and representations of racially and ethnically diverse students, faculty, and administrators in leadership positions; and involvement, retention, graduation, career choice, institutional racism, and campus conditions [5,12]. Moreover, these publications started to make recommendations for scholars, faculty, administrators, policy makers, and program managers to create more inclusive and diverse environments in higher education as more People of Color were given access to enter higher education institutions.

From 1976 to 1980, peer-reviewed journals in the United States mostly focused on minority students' characteristics, retention, and adjustment to campus life, in addition to suggesting what minority students "could do better to have a better college experience" ([12], p. 67). In the 1980s, scholarships began to change, as articles began to reflect a broader interest in, and portrayals of, racially 
and ethnically diverse students [12]. Students were seen as part of student leadership, involvement, volunteer work, and the use of facilities and resources, and eventually minority students were seen like the majority students who had to work through problems related to alcohol use and academic integrity issues. Banning et al. indicated that scholarship on racial and ethnic topics focused mostly on concerns related to Black students, staff, and faculty, and suggested that there was a need of scholarship with a focus on Hispanic/Latina/o, American Indian, and Asian American students, staff, and faculty [12]. Even though higher education academic peer-reviewed journals published some scholarly research on race and ethnicity, there was a lack of scholarship and research in academic peer-reviewed journals whose aims and scope focused on the production and dissemination of scholarship of race and ethnicity in American higher education.

\subsection{Conceptual Framework}

In this case study, I propose to engage all students, educators, practitioners, and administrators of higher education institutions in Boyer's Model of Scholarship, by creating a new peer-reviewed journal that aims to support and create opportunities to publish research, policy, best practices, and procedures with an emphasis on race and ethnicity in higher education. This case study was grounded in Boyer's Model of Scholarship, a model that promotes engagement in scholarship participation [25]. Boyer proposed an expended definition of scholarship based on four prominent approaches to understanding the importance of scholarship engagement: discovery, integration, application, and teaching. First, scholarship of discovery is the knowledge generated by conducting original research or creating other types of original work. This type of scholarship is measured by faculty performance on how much they publish in peer-reviewed forums, productivity, and performing creative work within established fields, and creating infrastructure for future studies [25].

Second, scholarship of integration involves the critical evaluation, synthesis, analysis, or interpretation of the research or creative work produced by others. Scholarship of integration is measured by the prepared comprehensive literature reviews, writing a textbook for use in multiple disciplines, and/or collaborating with colleagues to design and deliver a core course. Next, scholarship of application applies disciplinary expertise to the exploration and/or solution of institutional community, or social processes or problems. This type of scholarship is measured by serving institutions, industry, or government as a consultant; assuming leadership roles in professional organizations; and advising student leaders, thereby fostering professional growth [25]. Last, scholarship of teaching is the use of one's expertise to develop, transform, and extend teaching activities and other aspects of pedagogy in new and more effective ways. It includes research and other creative work that focuses on the improvement of teaching and learning. Scholarship of teaching is measured by the advancement of bridging theory and practice through research, developing, and testing instructional materials, mentoring graduate students, and designing and implementing a program-level assessment system [25].

Faculty members are rewarded based on publication [17], service, and teaching [19]. In order to be a "good" faculty member at a college or university, one must practice Boyer's four approaches to scholarship: discovery, integration, application, and teaching [25]. The purpose of scholarship and research is to make meaning and sense of a study, translate the findings into culturally accepted explanations [26], and present the results to a community engaged in the scholarship [27]. Research findings can be presented through publications, teaching, serving in committees, and a presentation to the community at a conference.

\section{Methods}

The case study approach has been utilized in research to understand complex issues and build upon information those in the field already know [28,29]. There are many critics who say this methodology cannot bring generalizability to the study and there are no grounds to offer reliability. However, Yin suggested that case study approach offers a solution with which to investigate real-life 
problems and offer reasonable solutions [28]. While there are many definitions of a case study, for this research project, I utilize Merriam and Tisdell's definition: "A case study is an in-depth description and analysis of a bounded system" ([29], p. 37).

Yin discussed four times when a case study design should be utilized [28]. First, the case study design should be utilized when the focus of the study is to answer how and why questions. Second, the case study approach should be applied to ones' research when one cannot manipulate the behavior of those involved in the study. Third, the case study approach should be used when one wants to cover contextual conditions, because the research believes the conditions are relevant to the phenomenon under study. Lastly, the case study approach should be considered when the boundaries are not clear between the phenomenon and context. Moreover, a case study has seven different approaches researchers can utilize, including explanatory, exploratory, descriptive, multiple case studies, intrinsic, instrumental, and collective [30]. For this study, the exploratory approach was utilized, because there was no single set of outcomes the researcher was looking for when investigating this phenomena. In conclusion, while it appears to be a simple approach to research, case studies allow researchers to further investigate a phenomena and help fill in gaps in knowledge [28-30].

In this exploratory case study, survey data were used to analyze the interest of NCORE's participants regarding the development of a peer-reviewed journal that aims to publish innovative scholarship on race and ethnicity in higher education. In addition, using the survey data, a new qualitative study was developed. Saunders and Wohlegemuth wrote that survey data can be used to address new study questions [31]. Semi-structured interviews were conducted, as a basic form of inquiry and a primary way to investigate [32]. Creswell's six steps for analysis of qualitative data were used to examine how a peer-reviewed journal would support administrators and faculty's practice and research in American higher education [33].

\subsection{Case Study Site and Participants}

Over the past 30 years, NCORE has facilitated the conference and conversation on race and ethnicity at a national level, across the United States [34]. The first Annual National Conference of Race and Ethnicity in American Higher Education (NCORE) was initiated and launched by The Southwest Center of Human Relations Studies, University of Oklahoma in 1988. NCORE was founded to address the resurgence of racist incidents in higher education, the national demographics and their projected impacts, and to provide a national resource and multicultural forum to attract Black/African Americans, American Indians, Asian/Pacific Islanders, Hispanic/Latinas/os, and European Americans. The conference creates and sustains a comprehensive institutional change design to improve racial and ethnic relations, to expand and provide opportunities for educational access and success to historically marginalized and underrepresented populations [35,36].

The 2013 NCORE conference set new attendance and participation records, with a total of 2177 conference attendees [36]. According to conference participant evaluation, 65\% identified themselves as administrators (including Assistant/Associate Vice President, Diversity Officer, Assistant Dean, Associate Dean, Department Chair of Director, Administrator, and Professional Staff), $12 \%$ identified themselves as faculty, $2 \%$ identified themselves as graduate students, $7 \%$ identified themselves as undergraduate students, and 8\% identified themselves as "other" [36]. All 2177 NCORE conference participants were invited via e-mail to participate in the survey for this case study.

From those that participated in the survey, seven individuals self-indicated in the open-ended survey question from the survey that they were interested in participating in an interview for the qualitative part of this exploratory case study. The seven qualitative interviews were included in the methods of this case study to examine and provide more detailed examination to further understand their interests, attitudes, and opinions on the need to create a peer-reviewed journal. At the time of the survey, all seven participants were involved with NCORE and agreed to participate in the interviews, but later only five participants completed the interviews of this case study. Of the five participants, two of them indicated they were retired faculty and have been involved with NCORE for 27 years, 
and one indicated he was faculty and involved with NCORE for 11 years. Two of the participants are administrators and have been involved with NCORE for 26 years and for seven years. Using the survey and transcripts as original data, all names of people and institutions were given a pseudonym, but names and pseudonyms are not used in this case study, as requested by all participants.

\subsection{Data Collection}

In June 2013, an invitation to participate in this study was sent via e-mail to all NCORE 2013 conference participants. The invitation included a survey with a section of eight questions to assess the attitudes, opinions, and interests of NCORE participants on the need of a peer-reviewed journal that aims to support and create opportunities to publish research, policy, best practices, and procedures with an emphasis on race and ethnicity in American higher education. Five of the survey questions used a Likert-scale, two questions were checkbox, and one question was open-ended. The survey data was collected using NCORE's Adobe FormsCentral Online Form and Surveys.

Using the survey data collected from the NCORE conference, a new set of questions was developed to interview NCORE participants. From the open-ended question, seven NCORE participants self-indicated interest in participating in an interview for the study. All seven participants were invited to participate in an interview. Seven participants were contacted via e-mail, and later only five agreed to participate in the interview. In order to qualify for the interview, participants must have attended NCORE, earned a doctoral degree, and served as an administrator or a faculty member at a college or university in the United States. Each participant was interviewed once; each interview lasted approximately $60 \mathrm{~min}$. All interviews were conducted via speakerphone in a private room and recorded using a hand-held recording device. All data were transcribed, and the transcripts served as data sources for analysis.

\subsection{Data Analysis}

The existing data from the NCORE conference survey was entered in an Excel document. This existing data was analyzed to evaluate the interest of NCORE members and participants on having a peer-reviewed journal affiliated with NCORE. The last question of the survey was open-ended; the responses were coded and then used to generate themes. The themes provided recommendations for issues and topics related to race and ethnicity that respondents were interested in reading and/or publishing.

For the qualitative study, all data collected during the interviews was analyzed using Creswell's six steps for qualitative data analysis. The first step was to organize and prepare data for analysis and transcribe interviews [33]. Second, interviews were transcribed, then read and reviewed; patterns emerged that gave a general sense of the information and its meaning. The principle investigator looked at what the participants said in data collected through the interviews. Third, the data was coded. Fourth, the coding process was used to generate a description of the setting or people, as well as the categories of themes for analysis. Fifth, the description and themes were represented in the qualitative narrative. The last steps were to examine lessons learned and interpret the findings.

\subsection{Findings}

The results of this case study are presented in two parts: first, a summary of the results of the survey data, and second, a summary of the qualitative data.

\section{Survey Data}

The NCORE conference survey was sent to all NCORE 2013 participants; $28 \%(n=605)$ of the conference participants completed the survey. Of those who participated in the survey, 65\% were administrators, $12 \%$ were faculty, $2 \%$ were graduate students, $7 \%$ were undergraduate students, and $8 \%$ were classified as 'other'. The survey results included a section of eight questions that assess the attitudes and opinions of the interest of NCORE participants on the need of a peer-reviewed journal 
that aims to publish research on race and ethnicity in American higher education. The results of the first seven questions are shown by the percentage with a brief summary.

A new peer-reviewed journal that aims to publish research on race and ethnicity in American higher education has the potential of a large readership audience. The survey data illustrates that $80.73 \%$ of the respondents were "very likely" or "likely" interested in reading a peer-review journal that aims to promote scholarship and ideas of race and ethnicity in American higher education. Whereas $10.8 \%$ of the survey respondents were "neutral", $3.98 \%$ were "unlikely" or "very unlikely" to read a new peer-reviewed journal.

The next question focused on interest in publishing: $51.74 \%$ of the survey respondents were "very likely" or "likely" interested in publishing in scholarship that focuses on race and ethnicity in American higher education. Counterparts stated that $22.39 \%$ were neutral, $17.74 \%$ were "unlikely" or "very unlikely" to publish, and $8.13 \%$ did not respond. All the survey participants are not researchers; therefore, the results of this answer indicated that not everyone is interested in being published in a peer-reviewed journal. However, a new peer-reviewed journal on race and ethnicity would be an additional outlet with which to support and promote the scholarship of faculty whose research exclusively focuses on race and ethnicity in higher education.

Additionally, 39.81\% of the respondents were "very likely" and "likely" interested in serving on the editorial board, $31.01 \%$ were "unlikely" or "very unlikely", and $24.88 \%$ were neutral. Similar to the questions of serving on an editorial board, $48.32 \%$ of respondents were "very likely" or "likely" interested to serve as a member of the peer-review committee; $23.38 \%$ were unlikely or very unlikely, and $21.72 \%$ were neutral. While there can be different roles and responsibilities between being on an editorial board and serving as a member of the peer-review committee, survey participants indicated they are interested in serving in a leadership position.

Historically, most peer-reviewed journals have not been open-access [1]; $52.08 \%$ of the respondents agreed that if there was a peer-reviewed journal sponsored by NCORE it should be obtained at no cost, and $30.45 \%$ agreed that cost should be included on the conferences dues, whereas $8.48 \%$ of the respondents believed that access to the journal should be an option by paying an additional fee. Approximately half of the survey participants were interested in having an open-access journal. An open access journal would allow readers to obtain access to the journal for no financial cost, while other survey participants believed that a fee should be included with NCORE dues.

Lastly, survey participants indicated that $78.61 \%$ of them were "very likely" and "likely" interested in a peer-reviewed journal that aims to publish their research and educational practices on race and ethnicity in American higher education, whereas $12.77 \%$ of the participants were "neutral", $4.48 \%$ were "unlikely" or "very unlike", and 4.15\% did not respond to the question. It is very likely, by $78.61 \%$, that NCORE participants will support a new peer-reviewed journal that aims to publish scholarship on race and ethnicity in American higher education. Survey participants indicated that they will support a new journal by reading, submitting manuscripts for publication, obtaining a membership, and serving as peer-reviewers of the journal.

Based on the interest of NCORE participants as current and future readers, these findings support the need for a publication that promotes scholarship on race and ethnicity in American higher education. In addition, survey participants indicated the top three most important issues and topics that they would like to read and/or publish include (1) more about the history and current political implications of race and ethnicity; (2) institutional, societal, and individual privilege and oppression; and (3) the implication of policy for communities of colors (including the following: affirmative action and Title IX in higher education; intersectionality of politics and higher education and race and ethnicity; policy and advocacy on how to protect culturally educational courses; issues affecting students of color for higher education access, matriculation, graduation, and affordability of college; students' of color financial aid and indebtedness; Deferred Action for Childhood Arrivals (DACA); and race and crime). 
The three main themes of community, institution, and policy provide a framework with which to organize and structure topics and issues indicated by survey participants. An administrator suggested in the open-ended question that, "Ideally, everything presented at NCORE should be published so that we can spread the word and get the movement (and conversation) going." Creating a new outlet for publishing topics and issues of race and ethnicity in higher education would help promote the knowledge of race and ethnicity (Asian American, Pacific Islander, African American/Black, European American, Latino/a/x-Hispanic, Native American/American Indian/Alaska Native, and multi-racial and bi-racial). A new peer-review affiliated with NCORE would develop new opportunities for administrators, faculty, and students to connect with colleagues from across the country to create new research opportunities, to present at NCORE, and to publish their work.

Overall, results indicated that survey participants were highly interested in supporting a peer-reviewed journal affiliated with NCORE, either by reading, publishing, and serving in the editorial board or as a peer-review. However, $50.2 \%$ of the respondents believe that the journal should be accessed at no cost. Survey participants believe that a development of a new peer-review should be an open-access journal, as an opportunity to promote NCORE and continue to learn about issues related to race and ethnicity. Overall, the majority of the respondents agreed that there is a need to develop additional peer-review journal outlets that produce more publications related to race and ethnicity in higher education.

\subsection{Qualitative Data}

From the survey data, a new study of questions was developed to capture, through semi-formal interviews, the opinions of NCORE participants on having a peer-reviewed journal aimed at publishing research and educational practice on race and ethnicity in American higher education. Interviewed participants shared their opinions and ideas related to a new journal with the focus of race and ethnicity, and how a new peer-reviewed journal might support administrators and faculty members' research and educational practices.

Reports showed that majority of NCORE participants over the last 30 years have been mostly administrators [36-39]. It is assumed that a new peer-reviewed journal affiliated with NCORE would attract and increase faculty participation. In this study, all interviewed participants stated that a new journal is not only an opportunity for faculty, but it is also an opportunity for administrators and students to publish research. For example, one of the participants stated:

Most of the scholarship that you read in all of the journals published is done by professors. In this case, because we have so many staff members coming to NCORE, this would be an excellent place for staff members from colleges and universities, where they would have a place to go, you know, for publications in an area of interest. I suspect that most of the journal would be by faculty, but my point would be that this might be a place where it's easier for staff members who work in diversity to publish as well. And then, I would hope that it would be an avenue that would facilitate graduate students' research publications opportunities.

The development of a new journal would also support the NCORE conference planning committee's goals and recommendations, to prepare people of color for faculty positions and promote development, and an outlet to maintain their research agenda. Participants also reported that a new journal affiliated with NCORE would "be like the one-stop spot", to be able to find scholarship on race and ethnicity in higher education connecting research and practice with faculty, practitioners, and students.

A new journal would also provide new outlets in which to publish scholarship related to race and ethnicity in American higher education. As stated earlier, Race Ethnicity in Education is the only international journal that aims to publish scholarship on race and ethnicity in education. Two of the participants stated that it is challenging to find outlets to publish their scholarship. More specifically, one faculty participant said: 
My research is so focused on race that it's often difficult to find other outlets for publication. There is a journal like, Race Ethnicity in Education; it's a British journal. That's like education broadly, there is not an outlet for high education in particular in American higher education.

Another participant said:

My research connects with race and ethnicity ... what I am trying to provide is our ethnic groups in the United States with a broader perspective about ethnicity in Latin America ... My experiences as a scholar practitioner is that there are a few outlets for me to publish and they are very rigorous or difficult to publish, and if you are not faculty you might not be able to publish in those journals.

Two other participants also noted that there were limiting outlets for publishing. They recognized that current and future generations of scholars have fewer outlets/opportunities to publish their scholarship on race and ethnicity. Another of the participants said:

I think the problem that the younger generation of scholars perhaps may be having is that you have, or will have ... is some constriction and reduction in the number of journals that aims and scope is on race and ethnicity.

A participant recognized that in order to publish, various journals seek scholarship from those that have earned a doctorate. The participant stated that a new peer-reviewed journal with a focus on race and ethnicity in American higher education would develop a new outlet for scholars and practitioners to publish. He stated, "It would be very, very valuable for young academicians to have a (new peer-reviewed) journal affiliated with NCORE because they have so relatively few opportunities to publish in existing journals".

If a new journal was developed and affiliated with NCORE, participants of this study advised selecting an editorial board and reviewers associated with NCORE. They suggested that the journal should be published twice a year and available online. Additionally, all participants proposed different names for a new peer-review journal that is sponsored by NCORE. Participants also suggested that a new journal should aim to explore issues of race and ethnicity in American higher education and its intersectionality. One of the participants shared:

The purpose is to publish the cutting-edge work around race, and intersection of race and ethnicity in American higher education, to advance the field forward. To take chances by publishing more critical work, or maybe work that doesn't fit traditional disciplinary approaches to publications, or to research in general. I think that an NCORE journal could be a really important place to publish unique contributions to the field of race and ethnicity in American higher education.

All five participants indicated that race and ethnicity in American higher education, and its intersection, should be the focus of a peer-reviewed journal affiliated with NCORE. Further, they suggested that the journal should promote human relations, social justice, equity, and human rights, through education, research, advocacy, and collaboration with institutions, communities, and policy makers.

\section{Discussion}

The quality and quantity of race and ethnicity in American higher education research and its relationship to practice remains limited. Based on the literature review and data collected, there is a need and interest to create a new peer-reviewed journal that focuses on scholarship of race and ethnicity in American higher education. While this study took place in the United States, based on the literature review and findings of this study, there is a need for new peer-review journals that aim to publish scholarship on race and ethnicity across higher education systems in the world. 
One way to support the creation of a new peer-review journal is to disrupt the status quo culture in higher education, as mainstream and top-tier journals do not focus on topics of race and ethnicity. Also, a new peer-reviewed journal associated with NCORE would serve as an additional outlet for Faculty of Color to publish their scholarship, as it is more challenging for them to publish their scholarship that focuses on race, ethnicity, and gender inequities in mainstream journals. Additionally, a new peer-reviewed journal affiliated with NCORE would support the recruitment, retention, and promotion and tenure of Faculty of Color. Lastly, a new peer-reviewed journal would play an important and unique role in today's American educational system and society. The future of a peer-reviewed journal with aims and scope on race and ethnicity in American higher education would offer alternative scholarship to educational research, and it would add literature centered on race and ethnicity. A new journal could advance the master narratives and epistemologies that have not been representative of races and ethnicities other than White.

With an understanding that we live in a society in which racism and oppression continue to be present every day, and that most people have been exposed to discourse through educational settings and various media communication forms that recreate privilege and oppression [40], it is important to start the conversation to challenge the mainstream methods and methodologies that often forget to recognize and do not represent people of color. Some communities of people feel threated and sensitive to conversations about race and ethnicity. While these conversations occur in various forums, it appears that race, (ethnicity), and racism seems nonexistent ... is mired in misinformation and miscommunication ... (and) Instead of talking about racism, we deflect our conversation with vague references to "culture", "diversity", "cross-cultural effectiveness" ([40], p. 3).

\section{Conclusions and Developments}

Research dedicated to the advancement of the study of race, ethnicity, and sovereignty on social justice in higher education and its practical applications remains limited. From the 2013-2015, I surveyed and interviewed NCORE participants soliciting their interests in having a peer-reviewed, interdisciplinary journal affiliated with NCORE. Participants were overwhelmingly interested in reading and publishing in a journal affiliated with NCORE, one that supports, promotes, and enhances research, ideas, issues, concerns, and promising practices for scholars, students, administrators, and faculty. Toward this end, in 2015, the first Journal Committed to Social Change on Race and Ethnicity (JCSCORE) issue was published. Since then, only four issues have been published each year (each issue with four manuscripts).

JCSCORE's vision is to promote and continue conversations on race and ethnicity in American higher education that are often dismissed by higher education culture. JCSCORE's mission is to promote an exchange of ideas to transform lives, enhance learning, and improve human relations in higher education. JCSCORE welcomes high-quality, original, and innovative manuscripts addressing the interconnections of race, ethnicity, and sovereignty in American higher education policy, practice, and theory.

A new peer-reviewed journal, JCSCORE, is making an impact at local, state, and national levels. In particular, it is a new publishing outlet on American race and ethnicity for Faculty of Color, emerging scholars, practitioners, educators, community allies, and activists. JCSCORE is making a significant impact by expanding and promoting the higher education conversations on race and ethnicity. However, it is important to recognize that one peer-review journal will not change the support and experiences of Faculty of Color. This case study demonstrates that there is a need for more peer-reviewed journals that focus on race and ethnicity in higher education. It is important to inform and understand the relationship of race and ethnicity in higher education systems across the world; this could ensure "that a wide range of voices and perspectives can access the scholarly record, both as authors and as readers" ([16], p. 108).

Conflicts of Interest: The author declares no conflicts of interest. 


\section{References}

1. SCImago Journal \& Country Rank. Journal Ranking. 2013. Available online: https://www.scimagojr.com/ journalrank.php (accessed on 4 May 2015).

2. Directory of Open Access Journals. DOAJ Search. 2013. Available online: http://www.doaj.org/ (accessed on 4 May 2015).

3. Goodman, D.J. Promoting Diversity and Social Justice: Educating People from Privileged Groups; Routledge: New York, NY, USA, 2011.

4. Turner, C.S.V.; Myers, S.L., Jr.; Creswell, J.W. Exploring underrepresentation: The case of faculty of color in the Midwest. J. High. Educ. 1990, 70, 27-59.

5. Delgado Bernal, D.; Villalpando, O. An apartheid of knowledge in academia: The struggle over the "legitimate" knowledge of faculty of color. Equity Excell. Educ. 2002, 35, 169-180. [CrossRef]

6. Stanley, C.A. When counter narratives meet master narratives in the journal editor-review process. Educ. Res. 2007, 36, 14-24. [CrossRef]

7. Jayakumar, U.M.; Howard, T.C.; Allen, R.C.; Han, C.J. Racial privilege in the professoriate: An exploration of campus climate, retention, and satisfaction. J. High. Educ. 2009, 80, 539-563. [CrossRef]

8. Turner, C.S.V.; González, J.C.; Wood, J.L. Faculty of color in academe. What 20 years of literature tells us. J. Divers. High. Educ. 2008, 1, 139-168. [CrossRef]

9. Urrieta, L., Jr.; Méndez, L.; Rodríguez, E. "A moving target": A critical race analysis of Latina/o faculty experiences, perspectives, and reflections on the tenure and promotion process. Int. J. Qual. Stud. Educ. 2015, 28, 1149-1168. [CrossRef]

10. Padilla, A.M. Ethnic minority scholars, research, and mentoring: Current and future issues. Educ. Res. 1994, 23, 24-27. [CrossRef]

11. Villalpando, O.; Delgado Bernal, D. A Critical Race Theory Analysis of Barriers that Impede the Success of Faculty of Color. In The Racial Crisis in American Higher Education; Smith, W., Altback, P., Lomotey, K., Eds.; SUNY Press: New York, NY, USA, 2002.

12. Banning, J.H.; Ahuna, L.M.; Hughes, B.M. A study of the "NASPA Journal" (1967-1996): A 30-year reflection of scholarship in student affairs focusing on race and ethnicity. NASPA J. 2000, 38, 58-99. [CrossRef]

13. Antonio, A.L. Faculty of color reconsidered reassessing contributions to scholarship. J. High. Educ. 2002, 73, 582-603. [CrossRef]

14. Solomon, D.J. The role of peer review for scholarly journals in the information age. J. Electron. Publ. $2007,10$. [CrossRef]

15. Francis, M. Peer reviewers of academic journals: Who is responsible for our professional literature. Coll. Res. Libr. 2012, 74, 535-545. [CrossRef]

16. Inefuku, H.W.; Roh, C. Agents of diversity and social justice: Librarians and scholarly communication. In Open Access and the Future of Scholarly Communication: Policy and Infrastructure; Smith, K.L., Dickson, K.A., Eds.; Rowman \& Littlefield: Lanham, MD, USA, 2016; pp. 107-127.

17. Bray, N.J.; Major, C.H. Status of journals in the field of higher education. In Proceedings of the Annual Meeting of the Association for the Study of Higher Education, Jacksonville, FL, USA, 11 May-11 August 2008.

18. Davis, D.E.; Astin, H.S. Reputational standing in academe. J. High. Educ. 1987, 58, 261-275. [CrossRef]

19. Mindiola, T., Jr. Getting Tenure at the U. In The Leaning Ivory Tower; Latino Professors in American Universities; Chavez, P.C., Padilla, R.V., Eds.; SUNY Press: New York, NY, USA, 1995; pp. 29-51.

20. Corby, K. Constructing core journal lists: Mixing science and alchemy. Libr. Acad. 2003, 3, 207-217. [CrossRef]

21. Harley, D.; Acord, S.K.; Earl-Novell, S. Peer Review in Academic Promotion and Publishing: Its Means, Focus, and Future; Center for the studies of higher education: Berkeley, CA, USA, 2010.

22. Stuart, J. Hispanic with Academic Credential Find Themselves Stretched Thin with Many Projects. Diverse Issues in Higher Education. October 2013. Available online: http:/ / diverseeducation.com/article/56485/ (accessed on 4 June 2015).

23. Thomas, P.L.; Schmidt, R. All that jazz: Doing and writing CQR in a material world. In Critical Qualitative Research Reade; Steinberg, S.R., Cannella, G.S., Eds.; Peter Lang Publishing: New York, NY, USA, 2012.

24. Connell, R. Writing for Research: Advice on Principle and Practice. 2015. Available online: http://www. raewynconnell.net (accessed on 29 May 2018).

25. Boyer, E.L. Scholarship Reconsidered: Priorities of the Professoriate; Jossey-Bass: San Francisco, CA, USA, 1997. 
26. Brew, A.; Boud, D. Teaching and Research: Establish the Vital Link with Learning. High. Educ. 1995, 29, 261-273. [CrossRef]

27. Wenger, L.; Hawkins, L.; Seifer, S.D. Community-Engaged Scholarship: Critical Junctures in Research, Practice, and Policy. J. High. Educ. Outreach Engagem. 2012, 16, 171-181.

28. Yin, R.K. Case Study Research: Design and Methods, 3rd ed.; Sage: Thousand Oaks, CA, USA, 2003.

29. Merriam, B.S.; Tisdell, J.E. Qualitative Research: A Guide to Design and Implementation, 4th ed.; Jossey-Bass: San Francisco, CA, USA, 2016.

30. Baxter, P.; Jack, S. Qualitative case study methodology: Study design and implementation for novice researchers. Qual. Rep. 2008, 13, 544-559.

31. Saunders, K.; Wohlegemuth, D.R. Using existing database. In Assessment Methods for Student Affairs; Schuh, J.H., Associated, Eds.; Jossey-Bass: San Francisco, CA, USA, 2009.

32. Seidman, I. Interviewing as Qualitative Research: A Guide for Researchers in Education $\mathcal{E}$ the Social Sciences, 4th ed.; SAGE: Thousand Oaks, CA, USA, 2013.

33. Creswell, J.W. Research Design: Qualitative, Quantitative, and Mixed Methods Approaches; SAGE Publications: Thousand Oaks, CA, USA, 2014.

34. Busby, B.S.; Salinas, C., Jr.; Wong, K.; Biscoe, B. Letter from the Editors: A new beginning. J. Comm. Soc. Chang. Race Ethn. 2015, 1, 1-3.

35. NCORE. About. 2013. Available online: https://www.ncore.ou.edu/en/about/ (accessed on 30 May 2018).

36. Southwest Center of Human Relations Studies. NCORE 2013 Conference recap. In Proceedings of the National Conference on Race \& Ethnicity in American Higher Education, New Orleans, LA, USA, 28 May-2 June 2013.

37. Southwest Center of Human Relations Studies. NCORE 2009 general evaluation. In Proceedings of the National Conference on Race \& Ethnicity in American Higher Education. In Proceedings of the National Conference on Race \& Ethnicity in American Higher Education, San Diego, CA, USA, 26-30 May 2009.

38. Southwest Center of Human Relations Studies. NCORE 2011 general evaluation. In Proceedings of the National Conference on Race \& Ethnicity in American Higher Education, San Francisco, CA, USA, 31 May-4 June 2011.

39. Southwest Center of Human Relations Studies. NCORE 2012 general evaluation. In Proceedings of the National Conference on Race \& Ethnicity in American Higher Education, New York, NY, USA, 29 May-2 June 2012.

40. Katz, J.H. White Awareness: HANDBOOK for Anti-Racism Training, 2nd ed.; University of Oklahoma Press: Norman, OK, USA, 2003.

(C) 2018 by the author. Licensee MDPI, Basel, Switzerland. This article is an open access article distributed under the terms and conditions of the Creative Commons Attribution (CC BY) license (http://creativecommons.org/licenses/by/4.0/). 